ANNALES

POLONICI MATHEMATICI

$88.2(2006)$

\title{
Multiple positive solutions for a second order delay boundary value problem on the half-line
}

\author{
by K. G. Mavridis, Ch. G. Philos and \\ P. Ch. Tsamatos (Ioannina)
}

\begin{abstract}
Second order nonlinear delay differential equations are considered, and Krasnosel'ski1's fixed point theorem is used to establish a result on the existence of positive solutions of a boundary value problem on the half-line. This result can be used to guarantee the existence of multiple positive solutions. A specification of the result obtained to the case of second order nonlinear ordinary differential equations as well as to a particular case of second order nonlinear delay differential equations is also presented. The applicability of the main result is demonstrated by an example.
\end{abstract}

1. Introduction. Boundary value problems on unbounded intervals have many applications in physical problems. Such problems arise, e.g., in the study of linear elasticity, fluid flows and foundation engineering (see [1, $8,15]$ and the references therein). An interesting overview on unbounded interval problems, including real world examples, history and various methods of proving solvability, can be found in the recent book by Agarwal and O'Regan [1]. Boundary value problems on unbounded intervals concerning second order ordinary or delay differential equations are of specific interest in these applications.

For second order ordinary or delay differential equations, boundary value problems on the half-line are closely related to the problems of existence of global solutions on the half-line with prescribed asymptotic behavior. Recently, there is, in particular, a growing interest in positive solutions of such boundary value problems (see, for example, [5-6, 8, 13-22]).

For the basic theory of delay differential equations, the reader is referred to the books by Diekmann et al. [7], and Hale and Verduyn Lunel [10]. In

2000 Mathematics Subject Classification: Primary 34K10, 34B18, 34B40; Secondary $47 \mathrm{H} 10$.

Key words and phrases: delay differential equation, solution, positive solution, multiple solutions, boundary value problem on the half-line, cone, Krasnosel'skiı̌'s fixed point theorem. 
particular, as concerns initial value problems, we refer to the monograph by Lakshmikantham and Leela [12], while, regarding boundary value problems, we mention the monographs by Azbelev, Maksimov and Rakhmatullina [3], and Azbelev and Rakhmatullina [4].

This paper deals with the existence of (multiple) nonnegative solutions of a boundary value problem on the half-line for second order nonlinear delay (and, especially, ordinary) differential equations. The paper can be considered as a continuation of the authors' previous work [17], but in a different direction.

Throughout the paper, for any interval $J$ of the real line $\mathbb{R}$ and any subset $S$ of $\mathbb{R}$, by $C(J, S)$ we will denote the set of all continuous functions defined on $J$ and having values in $S$. Moreover, $r$ will be a nonnegative real number. Furthermore, if $t \in[0, \infty)$ and $\chi$ is a continuous real-valued function defined at least on $[t-r, t]$, the notation $\chi_{t}$ will be used for the function in $C([-r, 0], \mathbb{R})$ defined by

$$
\chi_{t}(\tau)=\chi(t+\tau) \quad \text { for }-r \leq \tau \leq 0 .
$$

Notice that $C([-r, 0], \mathbb{R})$ is a Banach space with the usual sup-norm

$$
\|\psi\|=\sup _{-r \leq \tau \leq 0}|\psi(\tau)| \quad \text { for } \psi \in C([-r, 0], \mathbb{R}) .
$$

Consider the second order nonlinear delay differential equation

$$
x^{\prime \prime}(t)+f\left(t, x_{t}\right)=0,
$$

where $f$ is a real-valued function defined on the set $[0, \infty) \times C([-r, 0], \mathbb{R})$, which satisfies the following Continuity Condition:

(C) $f\left(t, \chi_{t}\right)$ is continuous with respect to $t$ in $[0, \infty)$ for each given function $\chi$ in $C([-r, \infty), \mathbb{R})$.

Our interest is concentrated on solutions of (1.1) on the whole interval $[0, \infty)$. By a solution on $[0, \infty)$ of $(1.1)$, we mean a function $x$ in $C([-r, \infty), \mathbb{R})$ which is twice continuously differentiable on $[0, \infty)$ and satisfies (1.1) for all $t \geq 0$.

With the delay differential equation (1.1), one associates an initial condition of the form

$$
x_{0}=\phi,
$$

where $\phi$ in $C([-r, 0], \mathbb{R})$ is given. In what follows, it will be supposed that

$$
\phi(0)=0 .
$$

Also, together with (1.1), we impose a condition of the form

$$
\lim _{t \rightarrow \infty} x^{\prime}(t)=\xi
$$


where $\xi$ is a given real number. Note that (1.3) implies

$$
\lim _{t \rightarrow \infty} \frac{x(t)}{t}=\xi
$$

Equations (1.1)-(1.3) constitute a boundary value problem (BVP, for short) on the half-line. A solution of (1.1)-(1.3) is a solution $x$ on $[0, \infty)$ of the delay differential equation (1.1) which satisfies the conditions (1.2) and (1.3).

Consider now the special case of the second order nonlinear ordinary differential equation

$$
x^{\prime \prime}(t)+g(t, x(t))=0,
$$

where $g$ is a continuous real-valued function on $[0, \infty) \times \mathbb{R}$.

We confine our attention only to solutions of (1.4) on the whole interval $[0, \infty)$. With (1.4), we associate the initial condition

$$
x(0)=0
$$

as well as the condition (1.3). In this special case, the BVP (1.1)-(1.3) reduces to the BVP (1.4), (1.5), (1.3).

Equation (1.1) is of a very general form, which includes as particular cases the ordinary differential equation (1.4), equations with retarded arguments (such as equation (1.6) below), and several other types of functional integrodifferential equations, for example the equation

$$
x^{\prime \prime}(t)+g\left(t, \int_{-r}^{0} x(t+s) d s\right)=0 .
$$

Here we specify our results for the most familiar particular cases of equation (1.4) and equation (1.6) below.

Next, consider the second order nonlinear delay differential equation

$$
x^{\prime \prime}(t)+h\left(t, x\left(t-T_{1}(t)\right), \ldots, x\left(t-T_{m}(t)\right)\right)=0,
$$

where $m$ is a positive integer, $h$ is a continuous real-valued function on $[0, \infty) \times \mathbb{R}^{m}$, and $T_{j}(j=1, \ldots, m)$ are nonnegative continuous real-valued functions on $[0, \infty)$ with

$$
\max _{j=1, \ldots, m} \sup _{t \geq 0} T_{j}(t)=r .
$$

We are interested in solutions of $(1.6)$ on the whole interval $[0, \infty)$. The initial condition

$$
x(t)=\phi(t) \quad \text { for }-r \leq t \leq 0
$$

(which is an equivalent form of (1.2)) as well as the condition (1.3) are associated with the delay differential equation (1.6). 
If (1.1) is to be equivalent to (1.6), we must define $f(t, \psi)=h\left(t, \psi\left(-T_{1}(t)\right), \ldots, \psi\left(-T_{m}(t)\right)\right)$ for $(t, \psi) \in[0, \infty) \times C([-r, 0], \mathbb{R})$.

The following lemma (see [17]) gives a useful integral representation of solutions of (1.1)-(1.3), which will be used to obtain the main result of the paper.

Lemma. A function $x$ in $C([-r, \infty), \mathbb{R})$ is a solution of $(1.1)-(1.3)$ if and only if

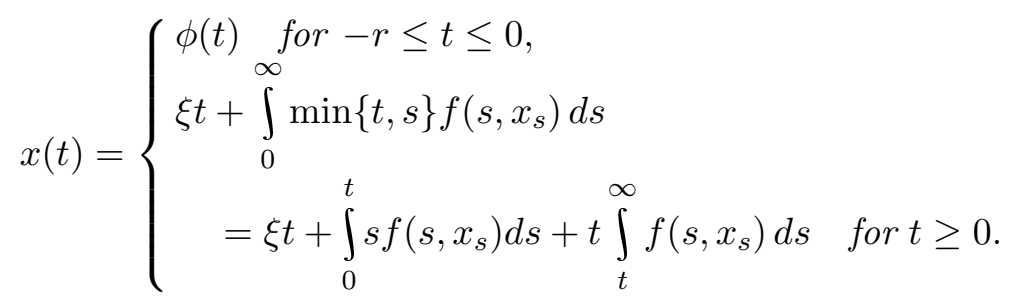

In [17], the authors studied the existence of solutions of (1.1)-(1.3) by using the classical Schauder fixed point theorem. Note that more general second order nonlinear delay differential equations, in which the nonlinear term involves the derivative of the unknown function, are also considered in [17]. In the present paper, we deal with the existence of nonnegative solutions of (1.1)-(1.3). It is noteworthy that the results given here can be used to guarantee the existence of multiple nonnegative solutions (see the example in Section 4). Our results in this paper are new even in the special case of ordinary differential equations.

Our purpose is to examine the existence of nonnegative solutions of (1.1)(1.3). So, without further mention, it will be assumed that

$$
\phi(t) \geq 0 \quad \text { for }-r \leq t \leq 0, \quad \text { and } \quad \xi \geq 0 .
$$

By the Lemma, every solution $x$ of (1.1)-(1.3) satisfies (1.7). Thus, we can easily arrive at the following simple result:

Assume that

$$
f(t, \psi) \geq 0 \quad \text { for all }(t, \psi) \in[0, \infty) \times C([-r, 0],[0, \infty)) .
$$

Then every nonnegative solution $x$ of (1.1)-(1.3) satisfies

$$
x(t) \geq \xi t \quad \text { for every } t \geq 0
$$

and

$$
x^{\prime}(t) \geq \xi \quad \text { for every } t \geq 0 .
$$

$[$ Because of $x(0)=\phi(0)=0,(1.9)$ is a consequence of (1.10).] Furthermore, every nonnegative solution $x$ of $(1.1)-(1.3)$ with $\xi>0$ is positive on $(0, \infty)$ with $\lim _{t \rightarrow \infty} x(t)=\infty$, and strictly increasing on $[0, \infty)$. 
The results of the paper are stated in Section 2. The main result gives sufficient conditions for (1.1)-(1.3) to have at least one nonnegative solution with a certain property. Two corollaries (Corollaries 1 and 2) are obtained by specifying the theorem to the case of the BVP (1.4), (1.5), (1.3) as well as to the particular case of the BVP (1.6), $\left(1.2^{\prime}\right),(1.3)$. Moreover, the theorem (specifically, Corollary 1 or 2 ) is applied to second order nonlinear (ordinary or delay) differential equations of Emden-Fowler type.

The proof of the theorem is given in Section 3.

The last section (Section 4) contains an example, which demonstrates the applicability of the results of the present paper.

2. Statement of the results. The main result of the paper is the following theorem, which provides sufficient conditions for (1.1)-(1.3) to have at least one nonnegative solution with a certain property.

Theorem. Suppose that (1.8) holds. Also, assume that, for each $t \geq 0$, the function $f(t, \cdot)$ is increasing on $C([-r, 0],[0, \infty))$ in the sense that $f(t, \psi) \leq f(t, \omega)$ for any $\psi, \omega$ in $C([-r, 0],[0, \infty))$ with $\psi \leq \omega(i . e ., \psi(\tau) \leq$ $\omega(\tau)$ for $-r \leq \tau \leq 0)$.

Assume that there exists a real number $c$ with $c \geq\|\phi\|$ and $c>\xi$ so that

$$
\int_{0}^{\infty} f\left(t, \eta_{t}\right) d t \leq c-\xi
$$

where $\eta \in C([-r, \infty),[0, \infty))$ depends on $c$ and is defined by

$$
\eta(t)= \begin{cases}c & \text { for }-r \leq t \leq 0, \\ c(t+1) & \text { for } t \geq 0 .\end{cases}
$$

Moreover, suppose that there exists a real number $b$ with $b>0$ and $b \neq c$ so that, for some fixed $t_{0}>0$,

$$
\int_{0}^{\infty} \min \left\{t_{0}, t\right\} f\left(t, \zeta_{t}\right) d t \geq b\left(t_{0}+1\right)-\xi t_{0},
$$

where $\zeta \in C([-r, \infty),[0, \infty))$ depends on $\phi, b$ and is defined by

$$
\zeta(t)= \begin{cases}\phi(t) & \text { for }-r \leq t \leq 0 \\ b \min \{t, 1\} & \text { for } t \geq 0\end{cases}
$$

Also, in the case where $b>c$, assume that

$$
\int_{0}^{\infty} f\left(t, \epsilon_{t}\right) d t<\infty
$$

where $\epsilon \in C([-r, \infty),[0, \infty))$ depends on $b$ and is defined by

$$
\epsilon(t)= \begin{cases}b & \text { for }-r \leq t \leq 0 \\ b(t+1) & \text { for } t \geq 0\end{cases}
$$


Then (1.1)-(1.3) has a nonnegative solution $x$ such that

$$
x(t) \geq\left[\sup _{T \geq 0} \frac{x(T)}{T+1}\right] \min \{t, 1\} \quad \text { for every } t \geq 0
$$

and

$$
\min \{c, b\} \leq \sup _{t \geq 0} \frac{x(t)}{t+1} \leq \max \{c, b\} .
$$

Notes. (i) Assume that $\xi>0$. Inequality (2.3) holds automatically if $b \leq \xi \frac{t_{0}}{t_{0}+1}$. (If $b \leq \xi \frac{t_{0}}{t_{0}+1}$, then $b<\xi$ and so $b<c$.)

(ii) Inequality (2.3) can equivalently be written as follows:

$$
\int_{0}^{t_{0}} t f\left(t, \zeta_{t}\right) d t+t_{0} \int_{t_{0}}^{\infty} f\left(t, \zeta_{t}\right) d t \geq b\left(t_{0}+1\right)-\xi t_{0} .
$$

(iii) From (2.7) and the first inequality of (2.8) it follows that the solution $x$ satisfies

$$
x(t) \geq \min \{c, b\} \min \{t, 1\} \quad \text { for every } t \geq 0 .
$$

By specifying our theorem to the case of the BVP (1.4), (1.5), (1.3), we get the following corollary.

Corollary 1. Suppose that

$$
g(t, y) \geq 0 \quad \text { for all } t \geq 0 \text { and } y \geq 0 .
$$

Also, assume that, for each $t \geq 0$, the function $g(t, \cdot)$ is increasing on $[0, \infty)$ in the sense that $g(t, y) \leq g(t, w)$ for any $y, w$ with $0 \leq y \leq w$.

Assume that there exists a real number $c$ with $c>\xi$ so that

$$
\int_{0}^{\infty} g(t, c(t+1)) d t \leq c-\xi .
$$

Moreover, suppose that there exists a real number $b$ with $b>0$ and $b \neq c$ so that, for some fixed $t_{0}>0$,

$$
\int_{0}^{\infty} \min \left\{t_{0}, t\right\} g(t, b \min \{t, 1\}) d t \geq b\left(t_{0}+1\right)-\xi t_{0} .
$$

Also, in the case where $b>c$, assume that

$$
\int_{0}^{\infty} g(t, b(t+1)) d t<\infty .
$$

Then the BVP (1.4), (1.5), (1.3) has a nonnegative solution $x$ such that (2.7) and (2.8) hold.

Similarly, specifying our theorem to the case of the BVP (1.6), (1.2'), (1.3) leads to the next corollary. 


\section{Corollary 2. Suppose that}

$$
h\left(t, y_{1}, \ldots, y_{m}\right) \geq 0 \quad \text { for all } t \geq 0 \text { and } y_{1} \geq 0, \ldots, y_{m} \geq 0 .
$$

Also, assume that, for each $t \geq 0$, the function $h(t, \cdot, \ldots, \cdot)$ is increasing on $[0, \infty)^{m}$ in the sense that $h\left(t, y_{1}, \ldots, y_{m}\right) \leq h\left(t, w_{1}, \ldots, w_{m}\right)$ for any $\left(y_{1}, \ldots, y_{m}\right),\left(w_{1}, \ldots, w_{m}\right)$ with $0 \leq y_{1} \leq w_{1}, \ldots, 0 \leq y_{m} \leq w_{m}$.

Assume that there exists a real number $c$ with $c \geq\|\phi\|$ and $c>\xi$ so that

$$
\int_{0}^{\infty} h\left(t, \varrho_{1}(t), \ldots, \varrho_{m}(t)\right) d t \leq c-\xi,
$$

where, for each $j \in\{1, \ldots, m\}$, the function $\varrho_{j}$ in $C([0, \infty),[0, \infty))$ depends on $c$ and is defined by

$$
\varrho_{j}(t)= \begin{cases}c & \text { for } 0 \leq t \leq T_{j}(t), \\ c\left(t-T_{j}(t)+1\right) & \text { for } t \geq T_{j}(t) .\end{cases}
$$

Moreover, suppose that there exists a real number $b$ with $b>0$ and $b \neq c$ so that, for some fixed $t_{0}>0$,

$$
\int_{0}^{\infty} \min \left\{t_{0}, t\right\} h\left(t, \sigma_{1}(t), \ldots, \sigma_{m}(t)\right) d t \geq b\left(t_{0}+1\right)-\xi t_{0},
$$

where, for each $j \in\{1, \ldots, m\}$, the function $\sigma_{j}$ in $C([0, \infty),[0, \infty))$ depends on $\phi, b$ and is defined by

$$
\sigma_{j}(t)= \begin{cases}\phi\left(t-T_{j}(t)\right) & \text { for } 0 \leq t \leq T_{j}(t) \\ b \min \left\{t-T_{j}(t), 1\right\} & \text { for } t \geq T_{j}(t) .\end{cases}
$$

Also, in the case where $b>c$, assume that

$$
\int_{0}^{\infty} h\left(t, \tau_{1}(t), \ldots, \tau_{m}(t)\right) d t<\infty
$$

where, for each $j \in\{1, \ldots, m\}$, the function $\tau_{j}$ in $C([0, \infty),[0, \infty))$ depends on $b$ and is defined by

$$
\tau_{j}(t)= \begin{cases}b & \text { for } 0 \leq t \leq T_{j}(t), \\ b\left(t-T_{j}(t)+1\right) & \text { for } t \geq T_{j}(t)\end{cases}
$$

Then the BVP (1.6), (1.2'), (1.3) has a nonnegative solution $x$ such that (2.7) and (2.8) hold.

To end this section, we will apply our theorem (or, specifically, Corollaries 1 and 2) to second order nonlinear (ordinary or delay) differential equations of Emden-Fowler type.

Consider the differential equations of Emden-Fowler type

$$
x^{\prime \prime}(t)+p(t)|x(t)|^{\gamma} \operatorname{sgn} x(t)=0
$$


and

$$
x^{\prime \prime}(t)+p(t)|x(t-r)|^{\gamma} \operatorname{sgn} x(t-r)=0,
$$

where $p$ is a nonnegative continuous real-valued function on $[0, \infty)$, and $\gamma$ is a positive real number.

For the BVP (2.9), (1.5), (1.3), our theorem (more specifically, Corollary 1 ) is formulated as follows:

Assume that there exists a real number $c$ with $c>\xi$ so that

$$
c^{\gamma} \int_{0}^{\infty}(t+1)^{\gamma} p(t) d t \leq c-\xi .
$$

Moreover, suppose that there exists a real number $b$ with $b>0$ and $b \neq c$ so that, for some fixed $t_{0}>0$,

$$
b^{\gamma} \int_{0}^{\infty} \min \left\{t_{0}, t\right\}(\min \{t, 1\})^{\gamma} p(t) d t \geq b\left(t_{0}+1\right)-\xi t_{0} .
$$

Then the BVP (2.9), (1.5), (1.3) has a nonnegative solution $x$ such that (2.7) and (2.8) hold.

Similarly, in the case of the BVP $(2.10),\left(1.2^{\prime}\right),(1.3)$, our theorem (more specifically, Corollary 2) leads to the following result:

Assume that there exists a real number $c$ with $c \geq\|\phi\|$ and $c>\xi$ so that

$$
c^{\gamma}\left[\int_{0}^{r} p(t) d t+\int_{r}^{\infty}(t-r+1)^{\gamma} p(t) d t\right] \leq c-\xi .
$$

Moreover, suppose that there exists a real number $b$ with $b>0$ and $b \neq c$ so that, for some fixed $t_{0}>0$,

$$
\begin{aligned}
\int_{0}^{r} \min \left\{t_{0}, t\right\} & {[\phi(t-r)]^{\gamma} p(t) d t } \\
& +b^{\gamma} \int_{r}^{\infty} \min \left\{t_{0}, t\right\}(\min \{t-r, 1\})^{\gamma} p(t) d t \geq b\left(t_{0}+1\right)-\xi t_{0} .
\end{aligned}
$$

Then the BVP (2.10), (1.2'), (1.3) has a nonnegative solution $x$ such that (2.7) and (2.8) hold.

3. Proof of the Theorem. The proof of our theorem is based on the use of the following Krasnosel'skiı fixed point theorem (see Krasnosel'skiı [11]; see also Guo and Lakshmikantham [9]).

The Krasnosel'SKII THEOREM. Let $E$ be a real Banach space endowed with the norm $\|\cdot\|_{E}$, and $K$ be a cone in E. Let also $\Omega_{1}$ and $\Omega_{2}$ be open 
bounded subsets of $E$ with $0 \in \Omega_{1}$ and $\bar{\Omega}_{1} \subset \Omega_{2}$. Assume that

$$
M: K \cap\left(\bar{\Omega}_{2} \backslash \Omega_{1}\right) \rightarrow K
$$

is a completely continuous mapping such that either

$$
\begin{array}{ll}
\|M y\|_{E} \leq\|y\|_{E} & \text { for } y \in K \cap \partial \Omega_{1}, \\
\|M y\|_{E} \geq\|y\|_{E} & \text { for } y \in K \cap \partial \Omega_{2},
\end{array}
$$

or

$$
\begin{aligned}
& \|M y\|_{E} \geq\|y\|_{E} \quad \text { for } y \in K \cap \partial \Omega_{1}, \\
& \|M y\|_{E} \leq\|y\|_{E} \quad \text { for } y \in K \cap \partial \Omega_{2} .
\end{aligned}
$$

Then the mapping $M$ has a fixed point (i.e., there exists $y \in K \cap\left(\bar{\Omega}_{2} \backslash \Omega_{1}\right)$ with $y=M y)$.

Let $B C([0, \infty), \mathbb{R})$ be the Banach space of all bounded continuous realvalued functions on $[0, \infty)$, endowed with the sup-norm $\|\cdot\|$.

We need the following compactness criterion for subsets of $B C([0, \infty), \mathbb{R})$, which is a consequence of the well-known Arzelà-Ascoli theorem (see the paper by Avramescu [2]). In order to formulate this criterion, we note that a set $U$ of real-valued functions defined on $[0, \infty)$ is called equiconvergent at $\infty$ if all functions in $U$ have finite limits at $\infty$ and, in addition, for each $\varepsilon>0$, there exists $T \equiv T(\epsilon)>0$ such that, for all functions $u$ in $U$, we have $\left|u(t)-\lim _{s \rightarrow \infty} u(s)\right|<\epsilon$ for every $t \geq T$.

COMPACTNESS CRITERION. Let $U$ be an equicontinuous and uniformly bounded subset of the Banach space $B C([0, \infty), \mathbb{R})$. If $U$ is equiconvergent at $\infty$, then it is relatively compact.

Throughout the remainder of this section, $E$ stands for the set of all functions $y$ in $C([0, \infty), \mathbb{R})$ with $y(t)=O(t)$ for $t \rightarrow \infty$. The set $E$ is a real Banach space endowed with the norm

$$
\|y\|_{E}=\sup _{t \geq 0} \frac{|y(t)|}{t+1} \quad \text { for } y \in E .
$$

Also, $P$ denotes the cone in $E$ consisting of all nonnegative functions $y$ in $E$ with $y(0)=0$. Moreover, for any function $y$ in $P$, we denote by $x$ the function in $C([-r, \infty),[0, \infty))$ defined by the formula

$$
x(t)= \begin{cases}\phi(t) & \text { for }-r \leq t \leq 0, \\ y(t) & \text { for } t \geq 0 .\end{cases}
$$

(Note that $\phi(t) \geq 0$ for $-r \leq t \leq 0$, and $\phi(0)=0$.)

In order to prove our theorem, we will first establish the following proposition.

Proposition. Suppose that (1.8) holds. Also, assume that, for each $t \geq 0$, the function $f(t, \cdot)$ is increasing on $C([-r, 0],[0, \infty))$. 
Let $d$ be a positive real number with $d \geq\|\phi\|$ such that

$$
\int_{0}^{\infty} f\left(t, \theta_{t}\right) d t<\infty,
$$

where $\theta \in C([-r, \infty),[0, \infty))$ depends on $d$ and is defined by

$$
\theta(t)= \begin{cases}d & \text { for }-r \leq t \leq 0 \\ d(t+1) & \text { for } t \geq 0\end{cases}
$$

Set

$$
\Omega=\left\{y \in E:\|y\|_{E}<d\right\} .
$$

Then the formula

$$
\begin{aligned}
(M y)(t) & =\xi t+\int_{0}^{\infty} \min \{t, s\} f\left(s, x_{s}\right) d s \\
& =\xi t+\int_{0}^{t} s f\left(s, x_{s}\right) d s+t \int_{t}^{\infty} f\left(s, x_{s}\right) d s \quad \text { for } t \geq 0
\end{aligned}
$$

makes sense for any $y \in P \cap \bar{\Omega}$, and defines a completely continuous mapping $M$ of $P \cap \bar{\Omega}$ into $P$.

Proof. Let $y \in P \cap \bar{\Omega}$. Then

$$
0 \leq y(t) \leq d(t+1) \quad \text { for } t \geq 0 .
$$

As $d \geq\|\phi\|$, we have

$$
0 \leq \phi(t) \leq d \quad \text { for }-r \leq t \leq 0 .
$$

Thus, by taking into account (3.1) and (3.3), we find

$$
0 \leq x(t) \leq \theta(t) \quad \text { for every } t \geq-r
$$

and consequently

$$
0 \leq x_{t} \leq \theta_{t} \quad \text { for all } t \geq 0 .
$$

(Note that, in view of $\phi(0)=0=y(0), x$ is continuous on $[-r, \infty)$.) Hence, by using the assumption (1.8) as well as the hypothesis that, for each $t \geq 0$, the function $f(t, \cdot)$ is increasing on $C([-r, 0],[0, \infty))$, we obtain

$$
0 \leq f\left(t, x_{t}\right) \leq f\left(t, \theta_{t}\right) \quad \text { for every } t \geq 0 .
$$

From (3.2) and (3.5) it follows that

$$
0 \leq \int_{0}^{\infty} f\left(t, x_{t}\right) d t<\infty \quad \text { for each } y \in P \cap \bar{\Omega} .
$$

By (3.6), we immediately see that the formula (3.4) makes sense for any $y \in P \cap \bar{\Omega}$, and that it defines a mapping $M$ of $P \cap \bar{\Omega}$ into $C([0, \infty),[0, \infty))$. We will show that $M(P \cap \bar{\Omega}) \subseteq P$. We observe that, for any $y \in P \cap \bar{\Omega}$, 
$M y$ is nonnegative on $[0, \infty)$, and $(M y)(0)=0$. Furthermore, by (3.5) and (3.2), for any $y \in P \cap \bar{\Omega}$ and $t \geq 0$, we obtain

$$
\begin{aligned}
\frac{(M y)(t)}{t+1} & =\xi \frac{t}{t+1}+\int_{0}^{\infty} \frac{\min \{t, s\}}{t+1} f\left(s, x_{s}\right) d s \\
& \leq \xi+\int_{0}^{\infty} f\left(s, x_{s}\right) d s \leq \xi+\int_{0}^{\infty} f\left(s, \theta_{s}\right) d s .
\end{aligned}
$$

Thus, we have

$$
\frac{(M y)(t)}{t+1} \leq N \quad \text { for every } t \geq 0
$$

where

$$
N=\xi+\int_{0}^{\infty} f\left(s, \theta_{s}\right) d s .
$$

Because of (1.8) and (3.2), $N$ is a nonnegative real number. We have thus proved that, for any $y$ in $P \cap \bar{\Omega}, M y \in P$, i.e., $M(P \cap \bar{\Omega}) \subseteq P$.

Now, we prove that $M(P \cap \bar{\Omega})$ is relatively compact. By the definition of $\|\cdot\|_{E}$, it suffices to show that the set

$$
U=\left\{u: \text { there exists } y \in P \cap \bar{\Omega} \text { such that } u(t)=\frac{(M y)(t)}{t+1} \text { for } t \geq 0\right\}
$$

is relatively compact in the Banach space $B C([0, \infty), \mathbb{R})$. We first observe that, by (3.7), $U$ is uniformly bounded. Moreover, by (3.5), for any $y \in P \cap \bar{\Omega}$ and $t \geq 0$, we get

$$
\begin{aligned}
\left|\left[\frac{(M y)(t)}{t+1}\right]^{\prime}\right|= & \frac{\left|(t+1)(M y)^{\prime}(t)-(M y)(t)\right|}{(t+1)^{2}} \\
= & \frac{1}{(t+1)^{2}} \mid(t+1)\left[\xi+\int_{t}^{\infty} f\left(s, x_{s}\right) d s\right] \\
& -\left[\xi t+\int_{0}^{t} s f\left(s, x_{s}\right) d s+t \int_{t}^{\infty} f\left(s, x_{s}\right) d s\right] \mid \\
= & \frac{1}{(t+1)^{2}}\left|\xi-\int_{0}^{t} s f\left(s, x_{s}\right) d s+\int_{t}^{\infty} f\left(s, x_{s}\right) d s\right| \\
\leq & \frac{1}{(t+1)^{2}}\left[\xi+\int_{0}^{t} s f\left(s, x_{s}\right) d s+\int_{t}^{\infty} f\left(s, x_{s}\right) d s\right] \\
= & \frac{\xi}{(t+1)^{2}}+\frac{1}{t+1} \int_{0}^{t} \frac{s}{t+1} f\left(s, x_{s}\right) d s+\frac{1}{(t+1)^{2}} \int_{t}^{\infty} f\left(s, x_{s}\right) d s
\end{aligned}
$$




$$
\begin{aligned}
& \leq \xi+\int_{0}^{t} f\left(s, x_{s}\right) d s+\int_{t}^{\infty} f\left(s, x_{s}\right) d s \\
& =\xi+\int_{0}^{\infty} f\left(s, x_{s}\right) d s \leq \xi+\int_{0}^{\infty} f\left(s, \theta_{s}\right) d s
\end{aligned}
$$

and consequently

$$
\left|\left[\frac{(M y)(t)}{t+1}\right]^{\prime}\right| \leq N \quad \text { for all } t \geq 0
$$

where the nonnegative real constant $N$ is defined by (3.8). In view of (3.9), we can apply the mean value theorem to conclude that

$$
\left|\frac{(M y)\left(t_{1}\right)}{t_{1}+1}-\frac{(M y)\left(t_{2}\right)}{t_{2}+1}\right| \leq N\left|t_{1}-t_{2}\right| \quad \text { for every } t_{1}, t_{2} \geq 0 .
$$

Hence $U$ is equicontinuous. Furthermore, by (3.5) again,

$$
\begin{aligned}
\left|\frac{(M y)(t)}{t+1}-\xi\right| & =\left|\frac{\xi t+\int_{0}^{\infty} \min \{t, s\} f\left(s, x_{s}\right) d s}{t+1}-\xi\right| \\
& =\frac{\left|-\xi+\int_{0}^{\infty} \min \{t, s\} f\left(s, x_{s}\right) d s\right|}{t+1} \\
& \leq \frac{\xi+\int_{0}^{\infty} \min \{t, s\} f\left(s, x_{s}\right) d s}{t+1} \\
& \leq \frac{\xi+\int_{0}^{\infty} \min \{t, s\} f\left(s, \theta_{s}\right) d s}{t+1}
\end{aligned}
$$

i.e.,

$$
\left|\frac{(M y)(t)}{t+1}-\xi\right| \leq \frac{\xi+\int_{0}^{\infty} \min \{t, s\} f\left(s, \theta_{s}\right) d s}{t+1} \quad \text { for all } t \geq 0
$$

But, by (3.2), we have

$$
\begin{aligned}
\lim _{t \rightarrow \infty} \frac{\xi+\int_{0}^{\infty} \min \{t, s\} f\left(s, \theta_{s}\right) d s}{t+1} & =\lim _{t \rightarrow \infty}\left[\xi+\int_{0}^{\infty} \min \{t, s\} f\left(s, \theta_{s}\right) d s\right]^{\prime} \\
& =\lim _{t \rightarrow \infty} \int_{t}^{\infty} f\left(s, \theta_{s}\right) d s=0
\end{aligned}
$$

Hence, because of (3.10), $U$ is equiconvergent at $\infty$. By the given compactness criterion, $U$ is relatively compact in $B C([0, \infty), \mathbb{R})$. So, the relative compactness of $M(P \cap \bar{\Omega})$ in $E$ has been established.

Next, we will show that the mapping $M$ is continuous. Let $\left(y^{[\nu]}\right)_{\nu \geq 1}$ be any sequence of functions in $P \cap \bar{\Omega}$ with

$$
\|\cdot\|_{E^{-}} \lim _{\nu \rightarrow \infty} y^{[\nu]}=y \in P \cap \bar{\Omega} .
$$


For each $\nu \geq 1$, we define

$$
x^{[\nu]}(t)= \begin{cases}\phi(t) & \text { for }-r \leq t \leq 0, \\ y^{[\nu]}(t) & \text { for } t \geq 0\end{cases}
$$

It is not difficult to verify that

$$
\|\cdot\|-\lim _{\nu \rightarrow \infty} x_{t}^{[\nu]}=x_{t} \quad \text { for every } t \geq 0 .
$$

On the other hand, by (3.5), we have

$$
0 \leq f\left(t, x_{t}^{[\nu]}\right) \leq f\left(t, \theta_{t}\right) \quad \text { for all } t \geq 0 \text { and } \nu \geq 1 .
$$

So, because of (3.2), we can apply the Lebesgue dominated convergence theorem to obtain, for $t \geq 0$,

$$
\lim _{\nu \rightarrow \infty} \int_{0}^{\infty} \min \{t, s\} f\left(s, x_{s}^{[\nu]}\right) d s=\int_{0}^{\infty} \min \{t, s\} f\left(s, x_{s}\right) d s .
$$

Thus, we have the pointwise convergence

$$
\lim _{\nu \rightarrow \infty}\left(M y^{[\nu]}\right)(t)=(M y)(t) \quad \text { for all } t \geq 0 .
$$

We shall also prove that

$$
\|\cdot\|_{E^{-}} \lim _{\nu \rightarrow \infty} M y^{[\nu]}=M y .
$$

For this, consider an arbitrary subsequence $\left(M y^{\left[\mu_{\nu}\right]}\right)_{\nu \geq 1}$ of $\left(M y^{[\nu]}\right)_{\nu \geq 1}$. As $M(P \cap \bar{\Omega})$ is relatively compact, there exists a subsequence $\left(M y^{\left[\mu_{\lambda_{\nu}}\right]}\right)_{\nu \geq 1}$ of $\left(M y^{\left[\mu_{\nu}\right]}\right)_{\nu \geq 1}$ such that

$$
\|\cdot\|_{E^{-}} \lim _{\nu \rightarrow \infty} M y^{\left[\mu_{\lambda_{\nu}}\right]}=u
$$

for some function $u$ in $E$. As $\|\cdot\|_{E}$-convergence implies pointwise convergence to the same limit function, we must have $u=M y$. This means that (3.11) holds true. Consequently, $M$ is continuous.

Since $M(P \cap \bar{\Omega})$ is relatively compact and $M$ is continuous, the mapping $M$ is completely continuous.

The proof of the proposition has been finished.

Now, we proceed to the proof of our theorem.

Proof of the Theorem. Set

$$
d=\max \{c, b\} .
$$

Clearly, $d$ is a positive real number with $d \geq\|\phi\|$. Let $\theta$ be the function in $C([-r, \infty),[0, \infty))$, which depends on $d$ and is defined by $(3.3)$. We see that

$$
\theta=\eta \quad \text { if } c>b, \quad \text { and } \theta=\epsilon \quad \text { if } b>c,
$$

where the functions $\eta$ and $\epsilon$ depend on $c$ and $b$, respectively, and are defined 
by (2.2) and (2.6), respectively. From (2.1) it follows, in particular, that

$$
\int_{0}^{\infty} f\left(t, \eta_{t}\right) d t<\infty .
$$

By taking into account (3.12) if $c>b$, and (2.5) if $b>c$, we conclude that (3.2) holds true. Set

$$
\Omega_{2}=\left\{y \in E:\|y\|_{E}<d\right\} .
$$

Then $\Omega_{2}$ is an open bounded subset of $E$. By our Proposition, the formula (3.4) makes sense for any $y \in P \cap \bar{\Omega}_{2}$, and defines a completely continuous mapping $M$ of $P \cap \bar{\Omega}_{2}$ into $P$.

We will show that

$$
\|M y\|_{E} \leq\|y\|_{E} \quad \text { for any } y \in P \cap \bar{\Omega}_{2} \text { with }\|y\|_{E}=c .
$$

Indeed, take any such $y$. Then

$$
0 \leq y(t) \leq c(t+1) \quad \text { for } t \geq 0 .
$$

Since $c \geq\|\phi\|$, we also have

$$
0 \leq \phi(t) \leq c \quad \text { for }-r \leq t \leq 0 .
$$

So, in view of (3.1) and (2.2),

$$
0 \leq x(t) \leq \eta(t) \quad \text { for all } t \geq-r,
$$

which yields

$$
0 \leq x_{t} \leq \eta_{t} \quad \text { for every } t \geq 0 .
$$

( $x$ is continuous on $[-r, \infty)$, since $\phi(0)=0=y(0)$.) Thus, by using (1.8) and the assumption that, for each $t \geq 0$, the function $f(t, \cdot)$ is increasing on $C([-r, 0],[0, \infty))$, we conclude that

$$
0 \leq f\left(t, x_{t}\right) \leq f\left(t, \eta_{t}\right) \quad \text { for all } t \geq 0 .
$$

Hence, for every $t \geq 0$, we obtain

$$
\begin{aligned}
\frac{(M y)(t)}{t+1} & =\xi \frac{t}{t+1}+\int_{0}^{\infty} \frac{\min \{t, s\}}{t+1} f\left(s, x_{s}\right) d s \\
& \leq \xi+\int_{0}^{\infty} f\left(s, x_{s}\right) d s \leq \xi+\int_{0}^{\infty} f\left(s, \eta_{s}\right) d s
\end{aligned}
$$

Consequently, by (2.1),

which gives

$$
\frac{(M y)(t)}{t+1} \leq c \quad \text { for every } t \geq 0
$$

$$
\|M y\|_{E}=\sup _{t \geq 0} \frac{(M y)(t)}{t+1} \leq c=\|y\|_{E}
$$

and so (3.13) has been proved. 
Now, we define

$$
K=\left\{y \in E: y(0)=0, \text { and } y(t) \geq \min \{t, 1\}\|y\|_{E} \text { for } t \geq 0\right\} .
$$

We immediately see that $K$ is a cone in $E$ with $K \subseteq P$. We shall prove that $M$ maps $P \cap \bar{\Omega}_{2}$ into $K$. Let $y \in P \cap \bar{\Omega}_{2}$. We observe that $(M y)(0)=0$. Next, it is easy to see that, for any $t, T \geq 0$,

$$
t \geq \begin{cases}\frac{t}{T+1} T & \text { for } 0 \leq t \leq 1 \\ \frac{1}{T+1} T & \text { for } t \geq 1\end{cases}
$$

That is,

$$
t \geq \frac{\min \{t, 1\}}{T+1} T \quad \text { for all } t, T \geq 0 .
$$

Moreover, it is not difficult to verify that, if $t, T, s \geq 0$, then

$$
\min \{t, s\} \geq \begin{cases}\frac{t}{T+1} \min \{T, s\} & \text { if } 0 \leq t \leq 1 \\ \frac{1}{T+1} \min \{T, s\} & \text { if } t \geq 1\end{cases}
$$

Hence

$$
\min \{t, s\} \geq \frac{\min \{t, 1\}}{T+1} \min \{T, s\} \quad \text { for all } t, T, s \geq 0 .
$$

By taking into account the hypothesis (1.8) and using (3.14) and (3.15), we obtain, for any $t, T \geq 0$,

$$
\begin{aligned}
(M y)(t) & =\xi t+\int_{0}^{\infty} \min \{t, s\} f\left(s, x_{s}\right) d s \\
& \geq \xi \frac{\min \{t, 1\}}{T+1} T+\frac{\min \{t, 1\}}{T+1} \int_{0}^{\infty} \min \{T, s\} f\left(s, x_{s}\right) d s \\
& =\min \{t, 1\}\left\{\frac{1}{T+1}\left[\xi T+\int_{0}^{\infty} \min \{T, s\} f\left(s, x_{s}\right) d s\right]\right\} \\
& =\min \{t, 1\} \frac{(M y)(T)}{T+1} .
\end{aligned}
$$

Therefore,

$$
(M y)(t) \geq \min \{t, 1\} \sup _{T \geq 0} \frac{(M y)(T)}{T+1} \quad \text { for all } t \geq 0,
$$

i.e.,

$$
(M y)(t) \geq \min \{t, 1\}\|M y\|_{E} \quad \text { for every } t \geq 0 .
$$

Thus $M y \in K$ for any $y \in P \cap \bar{\Omega}_{2}$. 
The next step is to show that

$$
\|M y\|_{E} \geq\|y\|_{E} \quad \text { for any } y \in K \cap \bar{\Omega}_{2} \text { with }\|y\|_{E}=b .
$$

For any such $y$,

$$
y(t) \geq \min \{t, 1\}\|y\|_{E} \quad \text { for } t \geq 0 .
$$

Consequently, because of $\|y\|_{E}=b$, we have

$$
y(t) \geq b \min \{t, 1\} \quad \text { for every } t \geq 0 .
$$

Hence, by taking into account (3.1) and (2.4), we obtain

$$
x(t) \geq \zeta(t) \geq 0 \quad \text { for all } t \geq-r,
$$

which gives

$$
x_{t} \geq \zeta_{t} \geq 0 \quad \text { for every } t \geq 0 .
$$

Thus, by using the hypothesis (1.8) as well as the assumption that, for each $t \geq 0$, the function $f(t, \cdot)$ is increasing on $C([-r, 0],[0, \infty))$, we get

$$
f\left(t, x_{t}\right) \geq f\left(t, \zeta_{t}\right) \geq 0 \quad \text { for } t \geq 0 .
$$

So, by the hypothesis (2.3), we derive

$$
\begin{aligned}
\sup _{t \geq 0} \frac{(M y)(t)}{t+1} \geq \frac{(M y)\left(t_{0}\right)}{t_{0}+1} & =\frac{1}{t_{0}+1}\left[\xi t_{0}+\int_{0}^{\infty} \min \left\{t_{0}, s\right\} f\left(s, x_{s}\right) d s\right] \\
& \geq \frac{1}{t_{0}+1}\left[\xi t_{0}+\int_{0}^{\infty} \min \left\{t_{0}, s\right\} f\left(s, \zeta_{s}\right) d s\right] \\
& \geq \frac{1}{t_{0}+1}\left\{\xi t_{0}+\left[b\left(t_{0}+1\right)-\xi t_{0}\right]\right\}=b,
\end{aligned}
$$

i.e.,

$$
\|M y\|_{E} \geq b=\|y\|_{E} .
$$

We have thus established (3.16).

Next, we put

$$
a=\min \{c, b\} .
$$

Clearly, $0<a<d$. Also, we consider the open bounded subset $\Omega_{1}$ of $E$ defined by

$$
\Omega_{1}=\left\{y \in E:\|y\|_{E}<a\right\} .
$$

It is obvious that the zero function in $E$ belongs to $\Omega_{1}$, and $\bar{\Omega}_{1} \subset \Omega_{2}$. By combining (3.13) and (3.16), it is easy to conclude that

$$
\begin{array}{ll}
\|M y\|_{E} \geq\|y\|_{E} & \text { for } y \in K \cap \partial \Omega_{1}, \\
\|M y\|_{E} \leq\|y\|_{E} & \text { for } y \in P \cap \partial \Omega_{2},
\end{array}
$$

if $c>b$, and

$$
\begin{array}{ll}
\|M y\|_{E} \leq\|y\|_{E} & \text { for } y \in P \cap \partial \Omega_{1}, \\
\|M y\|_{E} \geq\|y\|_{E} & \text { for } y \in K \cap \partial \Omega_{2},
\end{array}
$$


if $b>c$. That is, $M$ satisfies either (3.17) or (3.18). By the Krasnosel'skiu theorem, the restriction of $M$ to $K \cap\left(\bar{\Omega}_{2} \backslash \Omega_{1}\right)$ has a fixed point. So, there exists $y \in K \cap\left(\bar{\Omega}_{2} \backslash \Omega_{1}\right)$ with $y=M y$, i.e.,

$$
y(t)=\xi t+\int_{0}^{\infty} \min \{t, s\} f\left(s, x_{s}\right) d s \quad \text { for all } t \geq 0 .
$$

As $y \in K$, we have

$$
y(t) \geq \min \{t, 1\}\|y\|_{E} \quad \text { for every } t \geq 0 .
$$

Also, since $y \in \bar{\Omega}_{2} \backslash \Omega_{1}$,

$$
a \leq\|y\|_{E} \leq d .
$$

Now, because of (3.1) and (3.19), the function $x$ satisfies (1.7) and hence, by our lemma, $x$ is a nonnegative solution of (1.1)-(1.3). Furthermore, as $x \mid[0, \infty)=y$, we see that (3.20) and (3.21) coincide with (2.7) and (2.8), respectively.

The proof of the theorem is complete.

4. An example. Consider the Emden-Fowler differential equation

$$
x^{\prime \prime}(t)+p(t)[x(t)]^{2} \operatorname{sgn} x(t)=0,
$$

where $p$ is a nonnegative continuous real-valued function on $[0, \infty)$.

By applying our theorem or Corollary 1 (or, more specifically, the first of the two results stated in the last part of Section 2) to the BVP (4.1), (1.5), (1.3), we are led to the following result:

Assume that there exists a real number $c$ with $c>\xi$ so that

$$
c^{2} \int_{0}^{\infty}(t+1)^{2} p(t) d t \leq c-\xi .
$$

Moreover, suppose that there exists a real number $b$ with $b>0$ and $b \neq c$ so that, for some fixed $t_{0}>0$,

$$
b^{2} \int_{0}^{\infty} \min \left\{t_{0}, t\right\}(\min \{t, 1\})^{2} p(t) d t \geq b\left(t_{0}+1\right)-\xi t_{0} .
$$

Then the BVP (4.1), (1.5), (1.3) has a nonnegative solution $x$ such that (2.7) and (2.8) hold.

Now, set

$$
\xi=1, \quad p(t)=\frac{1}{36} e^{-t} \quad \text { for } t \geq 0 .
$$

Then it is a matter of elementary calculations to verify that (4.2) holds if and only if

$$
6 / 5 \leq c \leq 6
$$


(Note that $c$ must be greater than 1.) Furthermore, after some manipulations, we see that, if we choose $t_{0}=1$, then (4.3) is satisfied if and only if

$$
\text { either } 0<b \leq \frac{2\left[6-\sqrt{15\left(2+e^{-1}\right)}\right]}{2-5 e^{-1}} \quad \text { or } \quad b \geq \frac{2\left[6+\sqrt{15\left(2+e^{-1}\right)}\right]}{2-5 e^{-1}} .
$$

(We note that $b$ must be positive.) Take either

or

$$
c=\frac{6}{5} \quad \text { and } \quad b=\frac{2\left[6-\sqrt{15\left(2+e^{-1}\right)}\right]}{2-5 e^{-1}},
$$

$$
c=6 \quad \text { and } \quad b=\frac{2\left[6+\sqrt{15\left(2+e^{-1}\right)}\right]}{2-5 e^{-1}} .
$$

Then we conclude that:

The boundary value problem

$$
x^{\prime \prime}(t)+\frac{1}{36} e^{-t}[x(t)]^{2} \operatorname{sgn} x(t)=0, \quad x(0)=0, \quad \lim _{t \rightarrow \infty} x^{\prime}(t)=1,
$$

has two nonnegative solutions $x_{1}$ and $x_{2}$ such that

$$
x_{1}(t) \geq\left[\sup _{T \geq 0} \frac{x_{1}(T)}{T+1}\right] \min \{t, 1\}, \quad x_{2}(t) \geq\left[\sup _{T \geq 0} \frac{x_{2}(T)}{T+1}\right] \min \{t, 1\}
$$

for every $t \geq 0$, and

$$
\begin{aligned}
& \frac{2\left[6-\sqrt{15\left(2+e^{-1}\right)}\right]}{2-5 e^{-1}} \leq \sup _{t \geq 0} \frac{x_{1}(t)}{t+1} \leq \frac{6}{5}, \\
& 6 \leq \sup _{t \geq 0} \frac{x_{2}(t)}{t+1} \leq \frac{2\left[6+\sqrt{15\left(2+e^{-1}\right)}\right]}{2-5 e^{-1}} .
\end{aligned}
$$

Acknowledgments. The authors would like to thank the referee for his/her valuable suggestions on improving the paper.

\section{References}

[1] R. P. Agarwal and D. O'Regan, Infinite Interval Problems for Differential, Difference and Integral Equations, Kluwer, Dordrecht, 2001.

[2] C. Avramescu, Sur l'existence des solutions convergentes de systèmes d'équations différentielles non linéaires, Ann. Mat. Pura Appl. 81 (1969), 147-168.

[3] N. Azbelev, V. Maksimov and L. Rakhmatullina, Introduction to the Theory of Linear Functional-Differential Equations, Adv. Ser. Math. Sci. Engrg. 3, World Federation Publishers Company, Atlanta, GA, 1995.

[4] N. Azbelev and L. Rakhmatullina, Theory of Linear Abstract Functional-Differential Equations and Applications, Mem. Differential Equations Math. Phys. 8 (1996).

[5] C. Bai and J. Fang, On positive solutions of boundary value problems for secondorder functional differential equations on infinite intervals, J. Math. Anal. Appl. 282 (2003), 711-731. 
[6] A. Constantin, On the existence of positive solutions of second order differential equations, Ann. Mat. Pura Appl. 184 (2005), 131-138.

[7] O. Diekmann, S. A. van Gils, S. M. Verduyn Lunel and H.-O. Walther, Delay Equations: Functional, Complex, and Nonlinear Analysis, Springer, New York, 1995.

[8] D. Guo, Multiple positive solutions for first order nonlinear impulsive integro-differential equations in a Banach space, Appl. Math. Comput. 143 (2003), 233-249.

[9] D. J. Guo and V. Lakshmikantham, Nonlinear Problems in Abstract Cones, Academic Press, San Diego, 1988.

[10] J. K. Hale and S. M. Verduyn Lunel, Introduction to Functional Differential Equations, Springer, New York, 1993.

[11] M. A. Krasnosel'skiǔ, Positive Solutions of Operator Equations, Noordhoff, Groningen, 1964.

[12] V. Lakshmikantham and S. Leela, Differential and Integral Inequalities: Theory and Applications. Vol. II: Functional, Partial, Abstract, and Complex Differential Equations, Math. Sci. Engrg. 55-II, Academic Press, New York, 1969.

[13] Y. Liu, Boundary value problems for second order differential equations on unbounded domains in a Banach space, Appl. Math. Comput. 135 (2003), 569-583.

[14] - Existence and unboundedness of positive solutions for singular boundary value problems on half-line, ibid. 144 (2003), 543-556.

[15] —, Boundary value problems on half-line for functional differential equations with infinite delay in a Banach space, Nonlinear Anal. 52 (2003), 1695-1708.

[16] R. Ma, Existence of positive solutions for second-order boundary value problems on infinity intervals, Appl. Math. Lett. 16 (2003), 33-39.

[17] K. G. Mavridis, Ch. G. Philos and P. Ch. Tsamatos, Existence of solutions of a boundary value problem on the half-line to second order nonlinear delay differential equations, Arch. Math. (Basel), in press.

[18] K. G. Mavridis and P. Ch. Tsamatos, Positive solutions for first order nonlinear functional boundary value problems on infinite intervals, Electron. J. Qual. Theory Differ. Equ. 2004, no. 8, 18 pp.

[19] B. Yan, Multiple unbounded solutions of boundary value problems for second-order differential equations on the half-line, Nonlinear Anal. 51 (2002), 1031-1044.

[20] B. Yan and Y. Liu, Unbounded solutions of the singular boundary value problems for second order differential equations on the half-line, Appl. Math. Comput. 147 (2004), 629-644.

[21] Z. Yin, Monotone positive solutions of second-order nonlinear differential equations, Nonlinear Anal. 54 (2003), 391-403.

[22] Z. Zhao, Positive solutions of nonlinear second order ordinary differential equations, Proc. Amer. Math. Soc. 121 (1994), 465-469.

Department of Mathematics

University of Ioannina

P.O. Box 1186

45110 Ioannina, Greece

E-mail: kmavride@otenet.gr

cphilos@cc.uoi.gr

ptsamato@cc.uoi.gr

Received 14.11.2005

and in final form 2.1.2006

$(1646)$ 